\title{
Design and Synthesis of Novel Pyrrolo[2,1-c][1,4]benzodiazepine - Imidazole Containing Polyamide Conjugates
}

\author{
Rohtash Kumar, B.S.Narayan Reddy and J.William Lown* \\ Department of Chemistry, University of Alberta, Edmonton, AB, Canada, T6G 2G2
}

\begin{abstract}
A series of novel pyrrolo[2,1-c][1,4]benzodiazepine (PBD) - polyamide conjugates (1-5) containing imidazole units was synthesized as DNA minor groove bining agents.
\end{abstract}

\section{Introduction}

There is presently an interest in both the synthetic aspects and the mechanism of the biological action of the carbinolamine - containing pyrrolo[2,1-c][1,4]benzodiazepine (PBD) group of antitumor antibiotics, members of which include anthramycin, tomaymycin, neothramycins $A$ and $B$, sibiromycin, mazethramycin, chicamycin, prothracarcin DC-81, and dextrochrysin (1). They differ in the number, type, and position of the substituents in both there aromatic $\mathrm{A}$ - ring and pyrrolidine $\mathrm{C}$-rings, and in the degree of saturation of the $\mathrm{C}$ - ring, which can be either fully saturated or unsaturated at either $\mathrm{C} 2-\mathrm{C} 3$ (endocyclic) or $\mathrm{C} 2$ (exocyclic). A key feature of these molecules with respect to their mechanism of action is the N10-C11 carbinolamine (or imine equivalent) moiety. This is an electrophilic center responsible for alkylating DNA. After insertion in the minor groove, an aminal bond is formed through nucleophilic attack of the $\mathrm{N} 2$ of a guanine base at the electrophilic $\mathrm{C}-11$ position of PBD thus forming a covalent adduct in minor of DNA (2).

Most of the known natural PBDs have an (S) configuration at the chiral, C11, a position, which provides them with a right- handed twist when viewed from the $\mathrm{C}$ - ring towards to $\mathrm{A}$ - ring. This gives them the appropriate three dimensional shape for isohelicity with the minor groove of B-DNA, leading to a snug fit at the binding site. Racemization at $\mathrm{C} 11$ can significantly reduce both DNA - binding activity and in vitro cytotoxicity (3). Furthermore, the PBDs bind to DNA sequence selectively displaying a 
marked preference for 5 'PuGuPu sites (4) and have potential not only as antitumor agents but as gene regulaters and probes of DNA structure (5).

Recently, a C8-linked PBD dimer was prepared (6) which forms a symmetric interstrand cross- link with duplex DNA involving a four-base pair bonding site but spanning six DNA base pairs overall (7). Molecular modeling studies suggested that C 8 - linked PBD dimers have greater isohelicity with the minor groove of DNA (8) compared with the C7- linked dimers. Thurston et al. reported that the C8linked dimer DSB-120 ( Figure 1) forms an irreversible interstrand cross link between two guanine bases with in the minor groove via their exocyclic N2 atoms, and it spans six base pairs, actively recognizing a central 5'- GATC sequence (9). A variety of polyamide conjugates of other cytotoxic agents which are minor groove binders and with consequent improved selectivities and potencies have been reported in a review (10). Some efforts to date have been directed at different modifications on the PBD ring system with other well established DNA minor groove binders such as distamycin or netropsin (Figure 1). The latter agents bind to four or more consecutive A-T base pairs (11). In view of the commonly observed enhanced activity and selectivity of the parent drugs when conjugated with polyamides and the intrinsic activity of DSB-120, we have therefore attempted to conjugate certain polyamides with the PBD nucleous through the $\mathrm{C} 8$ - position with a suitable linker .

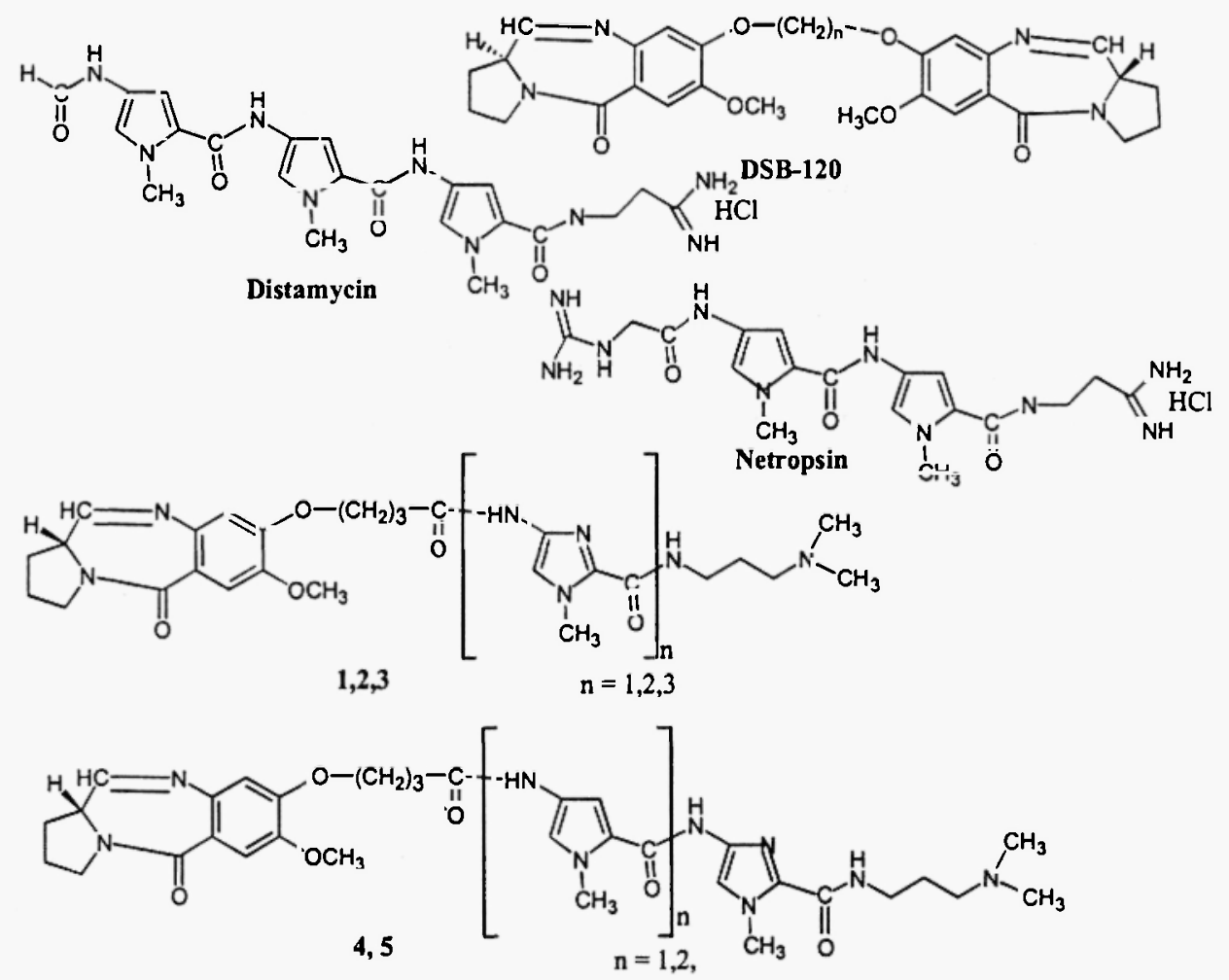

Figure -1 
In our group attempts have been made to link the PBD nucleus through the C8 position with a suitable linker to polyamides which contain pyrrole units (12). Studies on polyamides or information reading molecules show that replacement of pyrrole units by imidazoles cause a change in the base site recognition from AT to GC in the minor groove of DNA (13). In order to permit targeting of mixed.DNA sequences and to thereby investigate the effects of DNA sequence selective ability, we herein describe the synthesis of some PBD - polyamide conjugates, which contains imidazole units and pyrroles-imidazole units.

\section{Results and Discussion}

The compound 1- 5 were made according to the routes described in Scheme 1. The precursor of PBDnitro acid (14) was synthesized according to Scheme 1. The coupling of 5-methoxy-4- [3-(carboethoxy) propyloxy]-2-nitrobenzoic acid (8) via its acid chloride with (2S)- pyrrolidine-2carboxaldehydediethylthioacetal gave(2S)-N-[5-methoxy-4-[3-(carboethoxy) propyloxy]-2 nitrobenzoyl] pyrrolidine-2-carboxaldehydedietylthioacetal (9) in $75 \%$ yield which upon hydrolysis with IN $\mathrm{NaOH}$ produced the corresponding nitro acid (10). This latter was then coupled with the amine moiety of imidazoles containing polyamides with EDCI in dry DMF (15) in $70 \%$ yield. The corresponding amino compounds were then prepared by hydrogenation of corresponding nitro polyamides.

The nitro groups of compounds 11-15 were reduced with hydrogen in the presence of $\mathrm{Pd} / \mathrm{C}$ catalyst into their corresponding amino compounds which were then subjected to deprotective cyclization with $\mathrm{HgCl}_{2} / \mathrm{HgO}$ in aqueous acetonitrile at room temperature affording the corresponding imines $\mathbf{1 - 5}$ in $50 \%$ yield. Since the conjugation of the polyamides with PBD resulted in highly polar imines, this necessitated the use of methanol in combination with chloroform as eluent during the purification of the imines by column chromatography and therefore the product was obtained as a mixture of imine and its methyl ether in a 1: 1 ratio. The presence of both the forms was confirmed by both NMR and mass spectra. The final compounds were isolated in $35-40 \%$ yields.

In conclusion, in this communication we describe the synthesis of PBD-polyamide conjugates which contain imidazole units and pyrrole - imidazole units bonded through the C-8 position with a linker of three carbons. We have synthesized for the first time PBD- polyamide containing imidazoles units in order to examine their sequence- selective binding affinity with DNA. Efforts are under way to prepare complexes of these conjugates with duplex oligonucleotides for X-ray diffraction analysis, and the results will be disclosed in due course. 


\section{SCHEME 1}

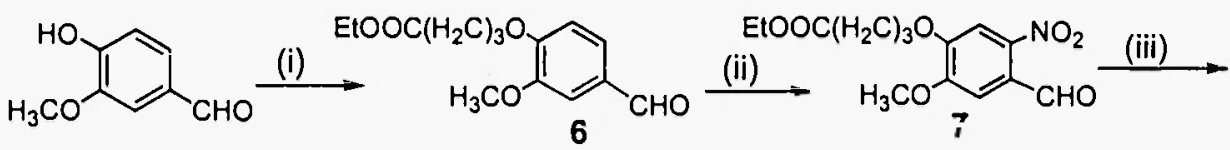

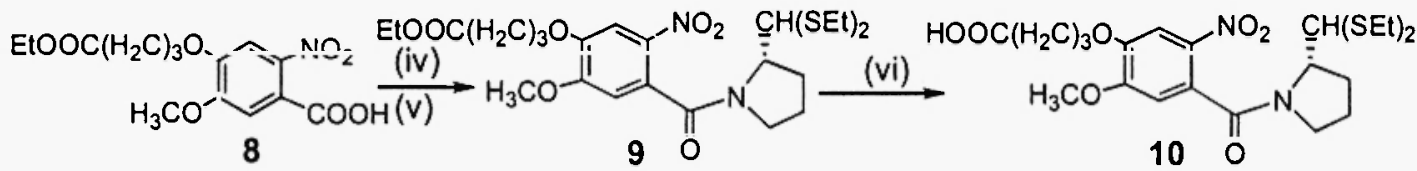

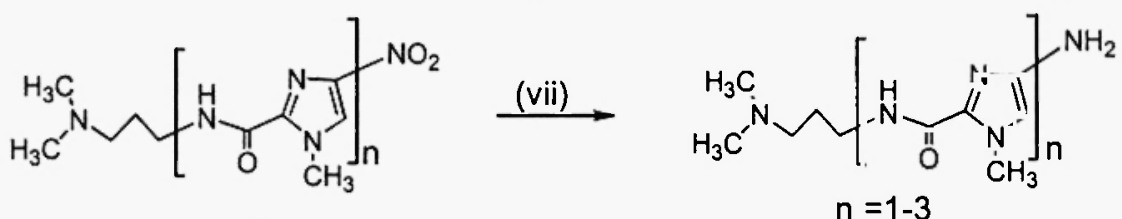

$n=1-3$

$\underset{(\text { viii })}{\stackrel{10}{\longrightarrow}}$

$11,12,13$

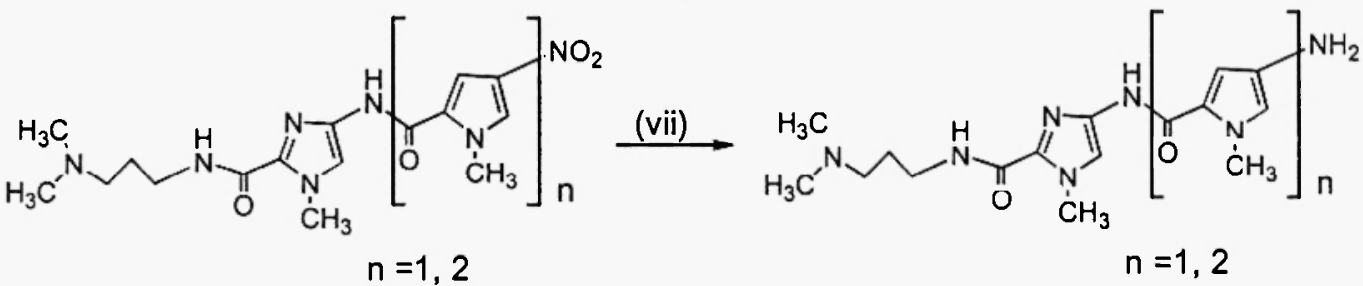

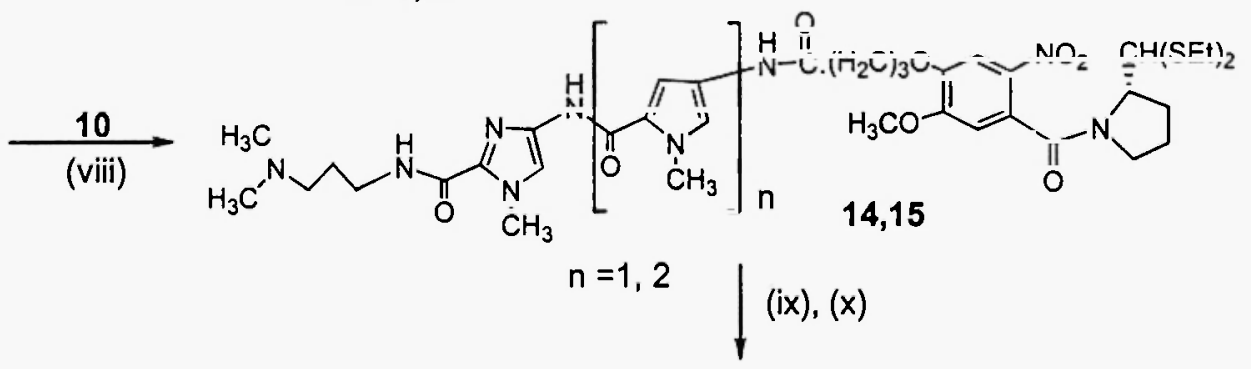

4,5

(i) $\mathrm{K}_{2} \mathrm{CO}_{3}, \mathrm{THF}, \mathrm{Br}\left(\mathrm{CH}_{2}\right)_{3} \mathrm{COOEt}$; (ii) $\mathrm{HNO}_{3} / \mathrm{SnCl}_{4}, \mathrm{DCM}$; (iii) sodium chlorite/sulfamic acid, $\mathrm{H}_{2} \mathrm{O}$; (iv) $\mathrm{SOCl}_{2} / \mathrm{C}_{6} \mathrm{H}_{6}, \mathrm{RT}, 2 \mathrm{~h}$.; (v) pyrrolidine-2-carboxaldehyde diethylthioacetal, $\mathrm{Et}_{3} \mathrm{~N}, \mathrm{DCM}, 0^{\circ} \mathrm{C}$, $1 \mathrm{~h}$.; (vi) $2 \mathrm{~N} \mathrm{NaOH}$ solution, $\mathrm{EtOH}, 50^{\circ} \mathrm{C}$, $18 \mathrm{~h}$; ; (vii) $\mathrm{H}_{2} / \mathrm{Pd}-\mathrm{C}, \mathrm{MeOH}$, 2h.; (viii) polyamide amine, HOBT, EDCl, dry DMF, RT, 14h.; (ix) $\mathrm{H}_{2} / \mathrm{Pd}-\mathrm{C}, \mathrm{MeOH}$; (x) $\mathrm{HgCl}_{2} / \mathrm{HgO}$, aq. $\mathrm{CH}_{3} \mathrm{CN}, \mathrm{RT}, \mathrm{Br}\left(\mathrm{CH}_{2}\right)_{3} \mathrm{COOEt}, 14 \mathrm{~h}$. 


\section{Experimental}

All the chemicals used were of reagent grade. Dimethylformamide (DMF) and methanol (MeOH) were of anhydrous grade procured from Aldrich Chemical Co. The 'H NMR spectra were recorded on a Bruker spectrometer (300MHz) . Mass spectra were determined on Associate Electrical Industries (AEI) MS-9 and MS-50 focussing high resolution mass spectrometers. Kieselgel 60 (230-400 mesh;E. Merck, Rahway, NJ) was used for flash column chromatography and precoated silica gel 60F-254 sheets (E. Merck) were used for thin layer chromatography. TLC plates were visualized by using UV light.

\section{General procedure for the synthesis of compounds 11-15}

A solution of nitropolyamides in $\mathrm{MeOH}$ was hydrogenated over $10 \% \mathrm{Pd} / \mathrm{C}$ at 50 psi pressure for two hours and then filtered through a Celite pad. The filtrate was concentrated to dryness under reduced pressure (at RT) to afford the corresponding amine. Owing to the sensitivity of the amine to oxidation, it was used for the next reaction immediately. It was dissolved in dry DMF and a mixture of the acid 10, hydroxybenzotriazole and EDCI in DMF was added. This mixture was stirred at RT for 12 hour and the solvent was removed under reduced pressure to afford a dark oil which was purified by flash column chromatography on silica gel by using methanol -dichloromethane as eluent to afford the coupled thioacetal $11-15$ as a yellow crystalline solid in $60 \%$ yield.

Compound 11: 'H NMR (DMSO- $\left.d_{6}, 300 \mathrm{MHz}\right): \delta 1.25(\mathrm{~m}, 6 \mathrm{H}), 1.71(\mathrm{~m}, 1 \mathrm{H}), 1.78(\mathrm{~m}, 2 \mathrm{H}), 1.91(\mathrm{~m}$, 2H), $2.05(\mathrm{t}, \mathrm{J}=6.9 \mathrm{~Hz}, 2 \mathrm{H}), 2.21(\mathrm{~m}, 1 \mathrm{H}), 2.55(\mathrm{~s}, 6 \mathrm{H}), 2.71(\mathrm{~m}, 4 \mathrm{H}), 2.95(\mathrm{~m}, 4 \mathrm{H}), 3.21-3.30(\mathrm{~m}, 4 \mathrm{H})$, $3.89(\mathrm{~s}, 3 \mathrm{H}), 3.93(\mathrm{~s}, 3 \mathrm{H}), 4.12(\mathrm{t}, \mathrm{J}=7.1 \mathrm{~Hz}, 2 \mathrm{H}), 4.50(\mathrm{~m}, 1 \mathrm{H}), 4.72(\mathrm{~d}, \mathrm{~J}=4.7 \mathrm{~Hz}, 1 \mathrm{H}), 6.91(\mathrm{~s}, 1 \mathrm{H})$, $7.40(\mathrm{~s}, 1 \mathrm{H}), 7.75(\mathrm{~s}, 1 \mathrm{H}), 8.45(\mathrm{t}, \mathrm{J}=6.5 \mathrm{~Hz}, 1 \mathrm{H}), 10.27(\mathrm{~s}, 1 \mathrm{H})$. HR-MS m/z calcd for $\mathrm{C}_{31} \mathrm{H}_{47} \mathrm{~N}_{7} \mathrm{O}_{7} \mathrm{~S}_{2}$ 693.31 , found $694.30(\mathrm{M}+1)$.

Compound 12: 'H NMR (DMSO- $\left.d_{6}, 300 \mathrm{MHz}\right): \delta 1.24(\mathrm{~m}, 6 \mathrm{H}), 1.70(\mathrm{~m}, 1 \mathrm{H}), 1.75-1.85(\mathrm{~m}, 2 \mathrm{H}), 1.89-$ $1.93(\mathrm{~m}, 2 \mathrm{H}), 2.06(\mathrm{t}, \mathrm{J}=6.5 \mathrm{~Hz}, 2 \mathrm{H}), 2.20(\mathrm{~m}, 1 \mathrm{H}), 2.56(\mathrm{~s}, 6 \mathrm{H}), 2.60-2.75(\mathrm{~m}, 4 \mathrm{H}), 2.90-2.95(\mathrm{~m}, 4 \mathrm{H})$, 3.21-3.35 (m, 4H), $3.81(\mathrm{~s}, 3 \mathrm{H}), 3.91(\mathrm{~s}, 3 \mathrm{H}), 3.95(\mathrm{~s}, 3 \mathrm{H}), 4.15(\mathrm{t}, \mathrm{J}=7.2 \mathrm{~Hz}, 2 \mathrm{H}), 4.50(\mathrm{~m}, 1 \mathrm{H}), 4.71(\mathrm{~d}$, $\mathrm{J}=4.5 \mathrm{~Hz}, 1 \mathrm{H}), 6.85(\mathrm{~s}, 1 \mathrm{H}), 7.50(\mathrm{~s}, 2 \mathrm{H}), 7.65(\mathrm{~s}, 1 \mathrm{H}), 8.45(\mathrm{t}, \mathrm{J}=6.2 \mathrm{~Hz}, 1 \mathrm{H}), 9.31(\mathrm{~s}, 1 \mathrm{H}), 10.41(\mathrm{~s}$, 1H). HR-MS $\mathrm{m} / \mathrm{z}$ calcd for $\mathrm{C}_{36} \mathrm{H}_{52} \mathrm{~N}_{10} \mathrm{O}_{8} \mathrm{~S}_{2} 816.31$, found $817.30(\mathrm{M}+1)$.

Compound 13: 'H NMR (DMSO- $\left.d_{6}, 300 \mathrm{MHz}\right): \delta 1.25(\mathrm{~m}, 6 \mathrm{H}), 1.61-1.70(\mathrm{~m}, 1 \mathrm{H}), 1.70-1.80(\mathrm{~m}, 2 \mathrm{H})$, 1.82-1.90 (m, 2H), $2.05(\mathrm{t}, \mathrm{J}=6.9 \mathrm{~Hz}, 2 \mathrm{H}), 2.21(\mathrm{~m}, 1 \mathrm{H}), 2.51(\mathrm{~s}, 6 \mathrm{H}), 2.60-2.75(\mathrm{~m}, 4 \mathrm{H}), 2.90-3.00(\mathrm{~m}$, $4 \mathrm{H}), 3.21-3.30(\mathrm{~m}, 4 \mathrm{H}), 3.81(\mathrm{~s}, 3 \mathrm{H}), 3.91(\mathrm{~s}, 3 \mathrm{H}), 3.93(\mathrm{~s}, 3 \mathrm{H}), 3.98(\mathrm{~s}, 3 \mathrm{H}), 4.15(\mathrm{t}, \mathrm{J}=7.2 \mathrm{~Hz}, 2 \mathrm{H})$, $4.51(\mathrm{~m}, 1 \mathrm{H}), 4.70(\mathrm{~d}, \mathrm{~J}=4.2 \mathrm{~Hz}, 1 \mathrm{H}), 6.85(\mathrm{~s}, 1 \mathrm{H}), 7.50(\mathrm{~s}, 2 \mathrm{H}), 7.64(\mathrm{~s}, 1 \mathrm{H}), 7.70(\mathrm{~s}, 1 \mathrm{H}), 8.45(\mathrm{t}, \mathrm{J}=$ $6.4 \mathrm{~Hz}, 1 \mathrm{H}), 9.61(\mathrm{~s}, 1 \mathrm{H}), 9.65(\mathrm{~s}, 1 \mathrm{H}), 10.5(\mathrm{~s}, 1 \mathrm{H})$. HR-MS m/z calcd for $\mathrm{C}_{41} \mathrm{H}_{57} \mathrm{~N}_{13} \mathrm{O}_{9} \mathrm{~S}_{2} 939.39$, found $940.39(\mathrm{M}+1)$. 
Compound 14: ${ }^{1} \mathrm{H}$ NMR (DMSO- $\left.d_{6}, 300 \mathrm{MHz}\right): \delta 1.25(\mathrm{~m}, 6 \mathrm{H}), 1.71(\mathrm{~m}, 1 \mathrm{H}), 1.78-1.85(\mathrm{~m}, 2 \mathrm{H}), 1.86-$ $1.95(\mathrm{~m}, 2 \mathrm{H}), 2.05(\mathrm{t}, \mathrm{J}=6.8 \mathrm{~Hz}, 2 \mathrm{H}), 2.15(\mathrm{~m}, 1 \mathrm{H}), 2.50(\mathrm{~s}, 6 \mathrm{H}), 2.60-2.76(\mathrm{~m}, 4 \mathrm{H}), 2.95-3.10(\mathrm{~m}, 4 \mathrm{H})$, 3.18-3.29 (m, 4H), $3.80(\mathrm{~s}, 3 \mathrm{H}), 3.81(\mathrm{~s}, 3 \mathrm{H}), 3.92(\mathrm{~s}, 3 \mathrm{H}), 4.16(\mathrm{t}, \mathrm{J}=7.2 \mathrm{~Hz}, 2 \mathrm{H}), 4.50(\mathrm{~m}, 1 \mathrm{H}), 4.72(\mathrm{~d}, \mathrm{~J}$ $=4.5 \mathrm{~Hz}, 1 \mathrm{H}), 6.89(\mathrm{~s}, 1 \mathrm{H}), 6.98(\mathrm{~d}, \mathrm{~J}=2.4 \mathrm{~Hz}, 1 \mathrm{H}), 7.21(\mathrm{~d}, \mathrm{~J}=2.4 \mathrm{~Hz}, 1 \mathrm{H}), 7.45(\mathrm{~s}, 1 \mathrm{H}), 7.70(\mathrm{~s}, 1 \mathrm{H})$, $8.20(\mathrm{t}, \mathrm{J}=6.5 \mathrm{~Hz}, 1 \mathrm{H}), 9.90(\mathrm{~s}, 1 \mathrm{H}), 10.35(\mathrm{~s}, 1 \mathrm{H})$. HR-MS m/z calcd for $\mathrm{C}_{37} \mathrm{H}_{53} \mathrm{~N}_{9} \mathrm{O}_{8} \mathrm{~S}_{2} 815.50$ found, $816.50(\mathrm{M}+1)$.

Compound 15: ${ }^{1} \mathrm{H}$ NMR (DMSO- $\left.d_{6}, 300 \mathrm{MHz}\right): \delta 1.24(\mathrm{~m}, 6 \mathrm{H}), 1.71(\mathrm{~m}, 1 \mathrm{H}), 1.85(\mathrm{~m}, 2 \mathrm{H}), 1.93(\mathrm{~m}$, $2 \mathrm{H}), 2.05(\mathrm{t}, \mathrm{J}=6.5 \mathrm{~Hz}, 2 \mathrm{H}), 2.18(\mathrm{~m}, 1 \mathrm{H}), 2.59(\mathrm{~s}, 6 \mathrm{H}), 2.65-2.75(\mathrm{~m}, 4 \mathrm{H}), 2.89-2.98(\mathrm{~m}, 4 \mathrm{H}), 3.30(\mathrm{~m}$, 4H), 3.81 (s, 3H), $3.84(\mathrm{~s}, 3 \mathrm{H}), 3.89(\mathrm{~s}, 3 \mathrm{H}), 3.93(\mathrm{~s}, 3 \mathrm{H}), 4.12(\mathrm{t}, \mathrm{J}=7.5 \mathrm{~Hz}, 2 \mathrm{H}), 4.51$ (m, 1H), 4.72 (d, J $=4.3 \mathrm{~Hz}, 1 \mathrm{H}), 6.83(\mathrm{~s}, 1 \mathrm{H}), 6.90(\mathrm{~d}, \mathrm{~J}=2.2 \mathrm{~Hz}, 1 \mathrm{H}), 7.06(\mathrm{~d}, \mathrm{~J}=2.2 \mathrm{~Hz}, 1 \mathrm{H}), 7.15(\mathrm{~d}, \mathrm{~J}=2.2 \mathrm{~Hz}, 1 \mathrm{H}), 7.30$ $(\mathrm{d}, \mathrm{J}=2.2 \mathrm{~Hz}, 1 \mathrm{H}), 7.49(\mathrm{~s}, 1 \mathrm{H}), 7.70(\mathrm{~s}, 1 \mathrm{H}), 8.23(\mathrm{t}, \mathrm{J}=6.5 \mathrm{~Hz}, 1 \mathrm{H}), 9.90(\mathrm{~s}, 1 \mathrm{H}), 9.92(\mathrm{~s}, 1 \mathrm{H}), 10.20(\mathrm{~s}$, 1H). HR-MS m/z calcd for $\mathrm{C}_{43} \mathrm{H}_{59} \mathrm{~N}_{11} \mathrm{O}_{8} \mathrm{~S}_{2} 937.40$, found $938.40(\mathrm{M}+1)$.

\section{General procedure for the synthesis of compounds 1-5}

To a solution of polyamide coupled thioacetals in methanol was added $10 \% \mathrm{Pd} / \mathrm{C}$ and the compound was hydrogenated in a par shaker at 50psi pressure for $2 \mathrm{hrs}$. TLC indicated the complete removal of starting material, and the reaction mixture was filtered on a small Celite ped to remove $\mathrm{Pd} / \mathrm{C}$ and then the filtrate was concentrated under reduced pressure to remove methanol completely. The resultant amino compound was dissolved in $\mathrm{CH}_{3} \mathrm{CN}-\mathrm{H}_{2} \mathrm{O}$ (4:1) and $\mathrm{HgCl}_{2}$ and $\mathrm{HgO}$ were added and the mixture was stirred slowly at RT for $12 \mathrm{~h}$. When TLC $\left(\mathrm{CHCl}_{3}-\mathrm{MeOH}\right.$-ammonia, 7:3:0.4) indicated the complete disappearance of the starting material, the reaction mixture was charged directly on to a short silica gel column and first eluted with ethyl acetate to remove $\mathrm{HgCl}_{2}$. After complete removal of $\mathrm{HgCl}_{2}$, the column was eluted with $\mathrm{CHCl}_{3}-\mathrm{MeOH}, 8: 2$, a system by which all other impurities were removed. Then the column was further eluted with $\mathrm{CHCl}_{3}-\mathrm{MeOH}$-ammonia, 7:3:0.1, and the ammonia concentration was slowly increased from 1 to $4 \%$. The imine compound was collected at $4 \%$ ammonia. The imine and the corresponding methyl ether move together as they have close $R_{f}$ values. Evaporation of the solvent under high vacuum at $\mathrm{RT}$ afforded an inseparable mixture of imine and methyl ether in an almost 1:1 ratio which is confirmed by its NMR data.

Compound 1: ' $\mathrm{H}$ NMR (DMSO- $\left.d_{6}, 300 \mathrm{MHz}\right): \delta 1.15-1.25(\mathrm{~m}, 2 \mathrm{H}) 1.61-1.80(\mathrm{~m}, 2 \mathrm{H}), 1.85-2.15(\mathrm{~m}$, $6 \mathrm{H}), 2.40(\mathrm{~s}, 6 \mathrm{H}), 2.56(\mathrm{~m}, 2 \mathrm{H}), 2.72(\mathrm{~m}, 1 \mathrm{H}), 3.21(\mathrm{~m}, 2 \mathrm{H}), 3.81-3.90(\mathrm{~m}, 7 \mathrm{H}), 3.91\left(\mathrm{~s}, 3 \mathrm{H}, \mathrm{ArOCH}_{3}\right)$, 3.93-4.10 (m, 3H, $\left.\mathrm{OCH}_{3}\right), 6.95(\mathrm{~s}, 1 \mathrm{H}), 7.10-7.50(\mathrm{~m}, 2 \mathrm{H}), 7.80(\mathrm{~d}, \mathrm{~J}=4.0 \mathrm{~Hz}, \mathrm{lH}$, imine proton), 8.10 $(\mathrm{m}, 1 \mathrm{H}), 10.40(\mathrm{~s}, 1 \mathrm{H})$. HR-MS $\mathrm{m} / \mathrm{z}$ calcd for $\mathrm{C}_{27} \mathrm{H}_{37} \mathrm{~N}_{7} \mathrm{O}_{5} 539.00$, found $540.30(\mathrm{M}+\mathrm{l})$ for imine compound and $572.30(\mathrm{M}+1)$ for its methyl ether compound. 
Compound 2: 'H NMR (DMSO- $\left.d_{6}, 300 \mathrm{MHz}\right): \delta 1.10-1.25(\mathrm{~m}, 2 \mathrm{H}), 1.75(\mathrm{~m}, 2 \mathrm{H}), 1.85-2.10(\mathrm{~m}, 6 \mathrm{H})$, $2.48(\mathrm{~s}, 6 \mathrm{H}), 2.56(\mathrm{~m}, 2 \mathrm{H}), 2.75(\mathrm{~m}, 1 \mathrm{H}), 3.21(\mathrm{~m}, 2 \mathrm{H}), 3.75-3.90(\mathrm{~m}, 10 \mathrm{H}), 3.92\left(\mathrm{~s}, 3 \mathrm{H}, \mathrm{ArOCH}_{3}\right), 3.93-$ $4.10\left(\mathrm{~m}, 3 \mathrm{H}, \mathrm{OCH}_{3}\right), 6.95(\mathrm{~s}, 1 \mathrm{H}), 7.10-7.50(\mathrm{~m}, 3 \mathrm{H}), 7.85(\mathrm{~d}, \mathrm{~J}=4.0 \mathrm{~Hz}, 1 \mathrm{H}$, imine proton), 8.40 $(\mathrm{m}, 1 \mathrm{H}), 9.41(\mathrm{~s}, 1 \mathrm{H}), 10.45(\mathrm{~s}, 1 \mathrm{H})$. HR-MS m/z calcd for $\mathrm{C}_{32} \mathrm{H}_{42} \mathrm{~N}_{10} \mathrm{O}_{6} 662.30$, found $663.33(\mathrm{M}+1)$ for imine compound and $695.36(\mathrm{M}+1)$ for its methyl ether compound.

Compound 3: 'H NMR (DMSO- $d_{6}, 300 \mathrm{MHz}$ ): $\delta$ 1.10-1.25 (m, 2H) 1.60-1.70 (m, 2H), 1.80-2.15 (m, $6 \mathrm{H}), 2.38(\mathrm{~s}, 6 \mathrm{H}), 2.48(\mathrm{~m}, 2 \mathrm{H}), 2.72(\mathrm{~m}, 1 \mathrm{H}), 3.20(\mathrm{~m}, 2 \mathrm{H}), 3.81-3.92(\mathrm{~m}, 13 \mathrm{H}), 3.93\left(\mathrm{~s}, 3 \mathrm{H}, \mathrm{ArOCH}_{3}\right)$, 3.95-4.10 (m, 3H, $\left.\mathrm{OCH}_{3}\right), 6.95-7.50(\mathrm{~m}, 5 \mathrm{H}), 7.70(\mathrm{~d}, \mathrm{~J}=4.0 \mathrm{~Hz}, 1 \mathrm{H}$, imine proton), $8.40(\mathrm{~m}, 1 \mathrm{H}), 9.70$ (brs, $2 \mathrm{H}$ ), $10.45(\mathrm{~s}, 1 \mathrm{H})$. HR-MS $\mathrm{m} / \mathrm{z}$ calcd for $\mathrm{C}_{37} \mathrm{H}_{47} \mathrm{~N}_{13} \mathrm{O}_{7} 785.30$, found $786.38(\mathrm{M}+1)$ for imine compound and $818.41(\mathrm{M}+1)$ for its methyl ether compound.

Compound 4: 'H NMR (DMSO- $\left.d_{6}, 300 \mathrm{MHz}\right): \delta 1.12-1.25(\mathrm{~m}, 2 \mathrm{H})$ 1.60-1.81 (m, 2H), 1.85-2.16 (m, $6 \mathrm{H}), 2.48(\mathrm{~s}, 6 \mathrm{H}), 2.56(\mathrm{~m}, 2 \mathrm{H}), 2.82(\mathrm{~m}, 1 \mathrm{H}), 3.25(\mathrm{~m}, 2 \mathrm{H}), 3.79-3.92(\mathrm{~m}, 10 \mathrm{H}), 3.93\left(\mathrm{~s}, 3 \mathrm{H}, \mathrm{ArOCH}_{3}\right)$, 3.98-4.10 (m, 3H, $\left.\mathrm{OCH}_{3}\right), 6.95(\mathrm{~s}, 1 \mathrm{H}) 7.10-7.60(\mathrm{~m}, 4 \mathrm{H}), 7.79(\mathrm{~d}, \mathrm{~J}=4.0 \mathrm{~Hz}, 1 \mathrm{H}$, imine proton), 8.20 $(\mathrm{m}, 1 \mathrm{H}), 9.41(\mathrm{~s}, 1 \mathrm{H}), 10.45(\mathrm{~s}, 1 \mathrm{H})$. HR-MS m/z calcd for $\mathrm{C}_{33} \mathrm{H}_{43} \mathrm{~N}_{9} \mathrm{O}_{6} 661.30$, found $662.30(\mathrm{M}+1)$ for imine compound and $694.30(\mathrm{M}+1)$ for its methyl ether compound.

Compound 5: 'H NMR (DMSO- $\left.d_{6}, 300 \mathrm{MHz}\right): \delta 1.12-1.25(\mathrm{~m}, 2 \mathrm{H}) 1.75(\mathrm{~m}, 2 \mathrm{H}), 1.95(\mathrm{~m}, 6 \mathrm{H}), 2.38(\mathrm{~s}$, $6 \mathrm{H}), 2.45(\mathrm{~m}, 2 \mathrm{H}), 2.75(\mathrm{~m}, 1 \mathrm{H}), 3.21(\mathrm{~m}, 2 \mathrm{H}), 3.85-3.94(\mathrm{~m}, 14 \mathrm{H}), 3.94\left(\mathrm{~s}, 3 \mathrm{H}, \mathrm{ArOCH}_{3}\right), 3.99(\mathrm{~m}, 3 \mathrm{H}$, $\left.\mathrm{OCH}_{3}\right), 6.95-7.60(\mathrm{~m}, 7 \mathrm{H}), 7.72(\mathrm{~d}, \mathrm{~J}=4.0 \mathrm{~Hz}, 1 \mathrm{H}$, imine proton), $8.45(\mathrm{~m}, 1 \mathrm{H}), 9.75$ (brs, $2 \mathrm{H}), 10.44(\mathrm{~s}$, 1H). HR-MS m/z calcd for $\mathrm{C}_{39} \mathrm{H}_{49} \mathrm{~N}_{11} \mathrm{O}_{7} 783.40$, found $784.40(\mathrm{M}+1)$ for imine compound and 816.40 $(\mathrm{M}+1)$ for its methyl ether compound.

Acknowledgement: The research was supported by a grant to J.W.Lown from the Natural Sciences and Engineering Research Council of Canada.

\section{References}

(1) Hurley, L.H.; Thurston, D.E. Pharm. Res. 52 (1984).; Hurley, L.H. J. Antibiot. 30, 349 (1977); Aoki, H.; Miyairi, N.; Ajisaka, M.;Sakai, H. J. Antibiot. 22, 201 (1969)

(2) Reddy, B.S.P.; Sondhi, S.M.; Lown, J.W. Pharmacology and Therapeutics. 84, 1 (1999)

(3) Miyamoto, M.; Kondo, S.; Naganawa, H.; Maeda, K.; Ohno, M.; Umezawa, H. J. Antibiot. 30, 340 (1977)

(4) Remers, W. Antitumor Antibiotics, Wiley and Sons, New York, PP $28-92$ (1988); Thurston, D.E. "Advances in the study of Pyrrolo[2,1-c][1,4]benzodiazepine (PBD Antitumor Antibiotics" in 
Molecular Aspects of Anticancer Drug DNA Interactions,Eds, Neidle, S. and Waring, M.J. Macmillan press Ltd. Pp 54-88 (1993)

(5) Hurley, L.H.; Reck, T.; Thurston, D.E.; Longley, D.R.; Holden, K. G.; Faucette, F. Jr.; Mong, S. M.; Johnson, R. K. Chem. Res. Toxicol. 1, 258 (1988)

(6) Bose, D. S.; Thompson, A. S.; Smellie, M.; Berardini, M. D.; Hartely, J. A.; Jenkins, T. C.; Neidle, S.; Thurston, D.E. J. Chem. Soc., Chem. Commun. 1518 (1992)

(7) Jenkins, T. C.; Hurley, L.H.; Neidle, S.; Thurston, D.E. J. Med. Chem. 37, 4529 (1994)

(8) Farmer, J. D.; Rudnicki, S. M. Jr.; Suggs, J. W. Tetrahedron Lett. 29, 5105 (1988)

(9) Bose, D. S.; Thompson, A. S.; Ching, J.; Hartely, J. A.; Berardini, M. D.; Jenkins, T. C.; Neidle, S.; Hurley, L.H.; Thurston, D.E. J. Am. Chem. Soc. 114, 4939 (1992)

(10) Sondhi, S. M.; Reddy, B. S. P.; Lown, J. W. Curr. Med. Chem. 4, 313 (1997)

(11) Lown, J. W.; Joshua, A. V. Biochemical Pharmacology 28, 2017 (1979)

(12) Damayanthi, Y.; Reddy, B. S. P.; Lown, J. W. J. Org. Chem. 64, 290 (1999)

(13) Rao, K. E.; Lown, J. W. Trends Org. Chem. 3, 141 (1992)

(14) Reddy, B. S. P.; Damayanthi, Y.; Reddy, B. S. N.; Lown, J. W. Anti-Cancer Drug Design. 15, 225 (2000)

(15) Gupta, R.; Xie, G.; Lown, J. W. Gene. 149, 81 (1994).; Gupta, R.; Wang, H.; Hung, L.; Lown, J. W. Anti-Cancer Drug Design. 10, 25 (1994)

Received on November 20, 2001 